\title{
Spotlight on emicizumab in the management of hemophilia A: patient selection and special considerations
}

This article was published in the following Dove Press journal:

Journal of Blood Medicine

\section{Koji Yada \\ Keiji Nogami}

Department of Pediatrics, Nara Medical University, Kashihara, Nara, Japan
Correspondence: Keiji Nogami Department of Pediatrics, Nara Medical University, 840 Shijo-cho, Kashihara, Nara 634-8522, Japan

Tel +8I 744298881

Fax +8I 744249222

Email roc-noga@naramed-u.ac.jp

\begin{abstract}
Emicizumab is a factor (F)VIIIa-mimicking bispecific antibody recognizing FIXa and FX molecules. In the phase 1/2 clinical studies, once-weekly subcutaneous administration of emicizumab has shown a favorable safety profile with encouraging efficacy in the patients with hemophilia A (PwHA) irrespective of the presence of anti-FVIII inhibitors. Moreover, in the following phase 3 studies, emicizumab treatment by once-weekly, biweekly or tetra-weekly administration have been still well-tolerated, but some thromboembolic events or thrombotic microangiopathy were observed associated with the concomitant use of activated prothrombin complex concentrates (aPCC) for breakthrough bleeds. Since approved for routine prophylaxis in PwHA in the US, EU, and Japan, a compass on patient selection for emicizumab treatment and special considerations on the practical situations such as concomitant treatment by bypassing agents (BPAs) or clotting factor concentrates (CFCs) with less thrombotic risk, inhibitor eradication by immune tolerance induction (ITI) should be provided. There is no doubt that emicizumab is an alternate first-line therapy for any existing BPA as hemostatic treatment for PwHA with inhibitor, but we should be more cautious in combination with aPCC on breakthrough bleeds under emicizumab prophylaxis because of thrombotic risk. For severe PwHA without inhibitor, since most patients are under CFCs prophylaxis, switching from CFCs to emicizumab should be considered when the advantage of emicizumab prophylaxis surpasses that of CFCs prophylaxis from the viewpoint of hemostatic effect by treatment, physical activity according to the life stage, health condition of the joints, adherence and complication. There are pros and cons on the timing of introduction of emicizumab for cases scheduled to start ITI or cases of ongoing ITI. Introduction of emicizumab to previously untreated patients and nonsevere PwHA without inhibitor is also required to discuss in consideration of risk of inhibitor development and unforeseen safety issues.
\end{abstract}

Keywords: hemophilia, emicizumab, inhibitor, noninhibitor, bypassing agent

\section{Introduction}

Hemophilia $(\mathrm{H}) \mathrm{A}$ is an X-chromosome linked congenital bleeding disorder caused by quantitative or qualitative defect of coagulation factor (F)VIII. There are three types of patients categorized as "severe", "moderate" and "mild" type based on base-line levels of procoagulant activity. ${ }^{1}$ Patients with HA (PwHA) present with serious hemorrhage such as articular and/or intramuscular bleeding from early childhood, and without appropriate treatment, repetitive joint bleedings lead to chronic synovitis, progressively resulting in irreversible hemophilic arthropathy. ${ }^{2,3}$ Hemarthrosis is controlled by advances with treatment using regular prophylaxis 
with clotting factor concentrates (CFCs), resulting in sufficient preventions of the onset of arthropathy and significant improvements in activity of daily living (ADL) and quality of life (QOL). ${ }^{4,5}$

However, under the current recommended therapy, there remain some significant barriers as follows. (i) The half-life of FVIII concentrates (8-12 hours) is so short that frequent intravenous infusion is required to prevent the repeated bleeding, which burdens a lot especially infant patients for whom venous access is very hard as well as their parents or caregivers. The requirement of frequent injection also causes low adherence to prophylaxis in adolescence. $^{6-8}$ (ii) Recently developed extended half-life (EHL)-FVIII products are currently available and reduce the frequency of intravenous administration. But treatment is still required twice per week (EHL type; $t_{1 / 2} \sim 1.5$-fold of FVIII). ${ }^{9-12}$ (iii) The development of anti-FVIII neutralizing alloantibodies (inhibitors) occurs in $20-30 \%$ of previously-untreated patients (PUPs) with HA after administration of FVIII concentrates. ${ }^{13,14}$ Bypassing agents (BPA) such as recombinant activated FVII (rFVIIa) and activated prothrombin complex concentrates (aPCC) are generally used as the hemostatic treatment for PwHA with high titer inhibitor. However, the hemostatic effect by BPA is not always sufficient, and several studies have shown that in rare cases, some PwHA with inhibitor are poorly responsive to bypassing agents after daily dosage. ${ }^{15,16}$ Immune tolerance induction (ITI) therapy is attempted to eliminate the inhibitor once developed for PwHA. ${ }^{15}$ ITI therapy is performed by infusion of FVIII concentrates with high dose and intensity, and $60-70 \%$ of PwHA with inhibitors are reported to be in remission after ITI therapy. ${ }^{16}$ However, this therapy burdens a lot of patients, because frequent infusion requires central venous access device (CVAD) in most cases and bypassing therapy is also needed on bleeding during ITI therapy. ${ }^{17}$

In order to overcome these unmet problems, novel therapeutic agents such as FVIII-mimicking bispecific antibody (emicizumab), ${ }^{18-24}$ anti-tissue factor pathway inhibitor antibody, ${ }^{25,26}$ and interference RNA inhibiting antithrombin $^{27}$ have been recently developed, and their safety and efficacy have been tested in the clinical trials. In this article, we highlight the patient selection and special considerations on management of PwHA by utilizing a novel hemostatic therapeutic, emicizumab (Hemlibra ${ }^{\circledR}$, Chugai/Roche) which offers the potential for a paradigm shift in the treatment of hemophilia, focusing on the issues surrounding the patients and their caregivers.

\section{Emicizumab}

\section{Functional properties}

The active form of FVIII (FVIIIa) enhances the interaction between FIXa (enzyme) and FX (substrate) by assigning the two coagulation factors to the spatially optimal positions on the phospholipid membrane surface, increasing the tenase activity by $\sim 10^{5}$-fold. ${ }^{28}$ Emicizumab is a humanized anti-FIXa/FX bispecific antibody categorized as human $\mathrm{IgG}_{4}$. In the course of emicizumab production, after 40,000 bispecific combinations have been screened and prototype antibodies of 2,400 variants were assessed, the lead antibody was maximally optimized to improve FVIIIa-mimetic activity, pharmacokinetics (PK), production, solubility, and to minimize immunogenicity. ${ }^{18,19}$ The epidermal growth factor (EGF)-like domain 1 of FIX/FIXa is recognized with one arm of emicizumab, while the EGF-like domain 2 of FX/FXa with another one. ${ }^{20}$ Therefore, emicizumab binds to FIXa and FX simultaneously and functions as a FVIIIa mimetic by mediating the appropriate structure in the tenase complex (Figure 1). As the emicizumab-binding affinity is weak, undesirable interference upstream and downstream of the coagulation cascade is minimized and antibody-dependent accumulation of $\operatorname{FIX(a)~and/or~FX(a)~in~circulation~is~not~induced~}$ by emicizumab. The simultaneous binding of emicizumab to FIXa and FX is necessary for its FVIIIa-mimicking function dependent on phospholipid surface. ${ }^{18}$ This finding indicates that emicizumab facilitates its activity only at the bleeding site, not in the normal circulation. The heavy chain of FVIII and emicizumab binds to FIXa and FX on phospholipid membrane, resulting in the enhancement of FVIIIa-mimicking effect. ${ }^{29}$ The FIXa-FX-emicizumab formation on phospholipid appears to mimic the conformation by the presence of the light chain of FVIII. ${ }^{29}$

Although emicizumab has similarities to FVIIIa, the results of laboratory tests should be carefully interpreted to evaluate the hemostatic efficacy, when comparing emicizumab with FVIIIa, in consideration of their difference. The effect of emicizumab is much more pronounced than native FVIII, as assessed by activated partial thromboplastin time (aPTT), ${ }^{18,20}$ because FVIII requires the time of activation step by thrombin, whilst emicizumab does not require this step. In terms of anti-coagulation action, emicizumab-driven 


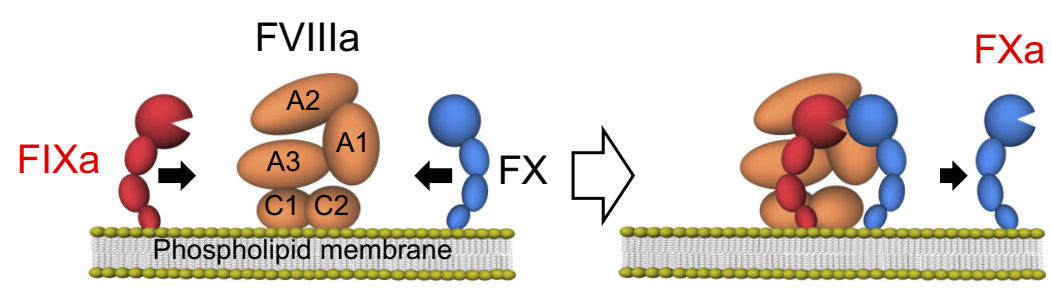

\section{Emicizumab}

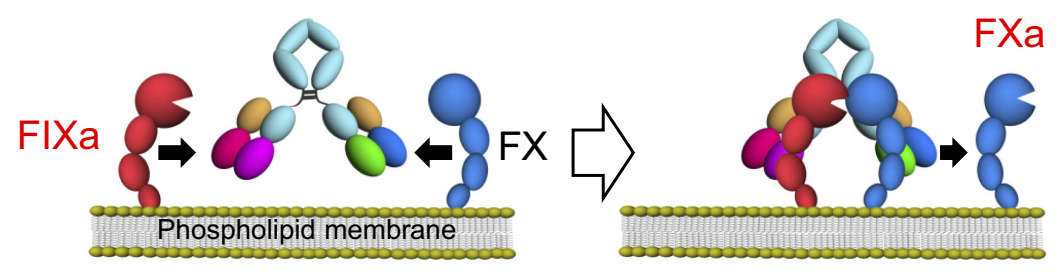

Figure I Mode of action of FVIIla or of a bispecific antibody, emicizumab.

Notes: FVIIla places FIXa and FX into spatially preferable position where FIXa efficiently catalyzes the activation of FX. Therefore, an asymmetric bispecific antibody that recognizes FIXa by one arm and FX by the other arm function as a FVIlla-mimetic cofactor.

Abbreviatons: FVIII, factor VIII; FVIIla, activated FVIII; FIXa, activated factor IX; FX, factor X.

thrombin generation is enough regulated by activated protein $\mathrm{C}$ through inactivation of FVa. ${ }^{30}$ In addition, the action on antithrombin of FIXa or FXa, or tissue factor pathway inhibitor on FXa is not interfered by emicizumab. ${ }^{31}$

\section{Clinical trials}

Phase I and I/2 study (ACE00IJP, ACE002JP study)

The phase 1 clinical study was conducted in 40 Japanese and 24 Caucasian healthy individual volunteers that received a single subcutaneous injection at a dose of emicizumab up to 1 $\mathrm{mg} / \mathrm{kg}$. Emicizumab demonstrated a linear PK pattern and $t_{1 / 2}$ of 4-5 weeks. ${ }^{21}$ The following "Patient part" was conducted in 18 Japanese severe HA patients with or without inhibitor ( $\geq 12$ years old; 11 inhibitors and 7 noninhibitors) using onceweekly subcutaneous injections of emicizumab at 0.3 (C-1 arm), 1 (C-2 arm) and $3 \mathrm{mg} / \mathrm{kg}$ (C-3 arm) for 12 weeks. $^{22}$ No serious adverse events (SAEs) were observed. No relevant clinical coagulation abnormalities or thromboembolic events were observed, although FVIII or BPAs was administered for breakthrough bleeds. Emicizumab did not influence plasma concentration levels of FIX and FX. The median annualized bleeding rates (ABRs) reduced from 32.5 to $4.4(\mathrm{C}-1)$, from 18.3 to 0.0 (C-2), and from 15.2 to 0.0 (C-3) significantly. No bleeding events were achieved in 8 inhibitor patients and in 5 noninhibitor patients (Table 1).

Sixteen patients continued to receive emicizumab in an extended study. ${ }^{23}$ A longer-term treatment ( $\leq 33$ months) with emicizumab was well-tolerated, with no development of neutralizing anti-emicizumab antibodies. No SAEs were observed. No thromboembolic events were observed in patients requiring concomitant therapy with FVIII or BPAs. Mean steady-state plasma trough levels of emicizumab were 10.3 (0.3 mg/kg; C-1), 29.9 (1 mg/kg; C-2) and 120 (3 mg/kg; C-3) $\mu \mathrm{g} / \mathrm{mL}$ dose proportionally. Median ABRs at $0.3,1$ and $3 \mathrm{mg} / \mathrm{kg}$ remained low at 1.4, 0.2 and 0 , relative to $4.4,0$ and 0 in the phase 1 study, respectively. No bleeding events were achieved in 8 patients. Dose uptitration resulted in a further reduction of $\mathrm{ABRs}$ in those patients with insufficient bleeding control, and the administration for FVIII or BPAs was significantly diminished.

\section{Phase 3 study (HAVEN I, 2 study)}

Following the phase $1 / 2$ studies, a global phase 3 study was conducted (HAVEN 1). ${ }^{24}$ A HAVEN 1 study evaluated subcutaneous emicizumab prophylaxis once-weekly for PwHA with inhibitors ( $\geq 12$ years old). The dosing schedule was determined by model-based simulation without dose escalation. ${ }^{32}$ Patients who have received episodic BPA were assigned in a ratio of $2: 1$ to emicizumab prophylaxis group $(\mathrm{N}=35)$ or no prophylaxis group $(\mathrm{N}=18)$. Patients who weretreated with prophylactic BPA were also assigned to emicizumab prophylaxis group $(\mathrm{N}=56)$. The primary endpoint was the difference in ABR between emicizumab treated and nontreated group. Emicizumab was administered at $3.0 \mathrm{mg} / \mathrm{kg}$ for fourweeks, followed by $1.5 \mathrm{mg} / \mathrm{kg}$ every week. The median ABR was 2.9 in emicizumab- 


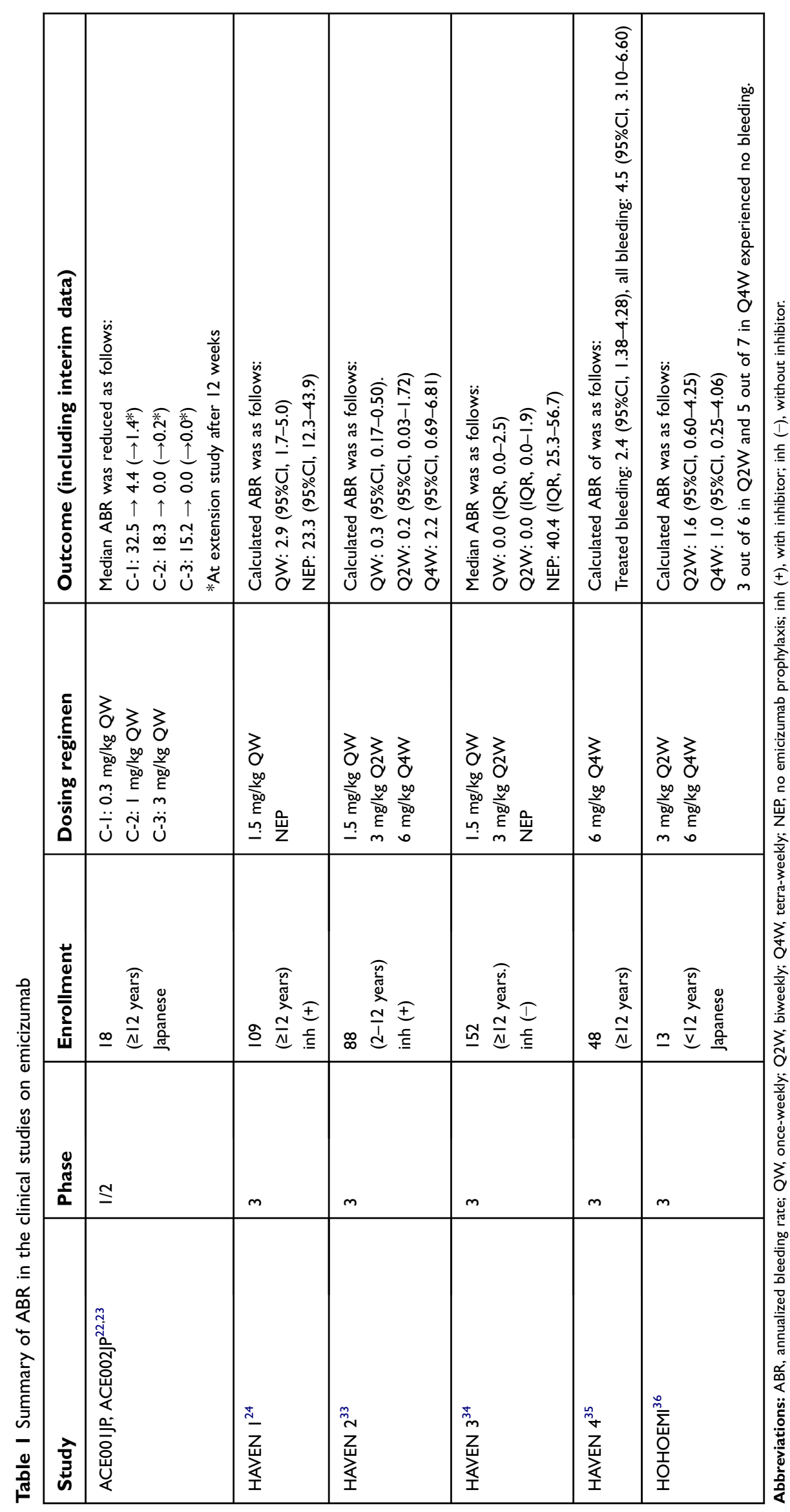


treated patients and 23.3 in nontreated patients, which indicates an $87 \%$ reduction in the emicizumab prophylaxis group. In addition, $63 \%$ in emicizumab-treated patients had no bleeding episode, whilst $6 \%$ in nontreated patients. Compared to the group previously receiving BPA prophylaxis, emicizumab prophylaxis evidently decreased the ABR (79\%). The most common treatment-related adverse events (AEs) were injection site reactions (15\%). Thrombotic microangiopathy (TMA; 3 cases) and thrombotic events (TE; 2 cases) occurred during concomitant therapy with emicizumab and aPCC (see "4)-i) Serious adverse event"). More recently, one case occurred TMA during concomitant therapy with aPCC has been reported.

Another phase 3 study (HAVEN 2) was established for pediatric patients with inhibitor. ${ }^{33}$ Eighty-eight HA children with inhibitors (2-12 years old) were enrolled, and an interim analysis demonstrated that $76.9 \%$ of patients did not require additional hemostatic treatment, whilst $23.1 \%$ experienced 1-3 treated bleeds. The ABRs were 0.3 in emicizumab-treated children. A remarkable decrease (99\%) in ABR was observed in emicizumab prophylaxis compared to previous BPA treatment. Significant improvements in health-related quality of life and burden on caregivers were seen. No thromboembolic events were observed. Average steady-state emicizumab concentrations of approximately $50 \mu \mathrm{g} / \mathrm{mL}$ were maintained. One case detected the neutralizing antidrug antibody (ADA), however. In summary, emicizumab prophylaxis produced a significantly lower bleeding rate in HA patients with inhibitor.

\section{Phase 3 study (HAVEN 3, 4 and HOHOEMI study)}

HAVEN 3 (NCT02847637) was conducted as a randomized open-labeled study for severe PwHA ( $\geq 12$ years old) without inhibitors. ${ }^{34}$ On-demand patients $(\mathrm{N}=89)$ were assigned into a 2:2:1 ratio to receive as a loading dose at $3 \mathrm{mg} / \mathrm{kg}$ weekly for four weeks, followed by $1.5 \mathrm{mg} / \mathrm{kg}$ weekly $(\mathrm{N}=36)$, or 3 $\mathrm{mg} / \mathrm{kg}$ bi-weekly $(\mathrm{N}=35)$ as long-term prophylaxis or no prophylaxis $(\mathrm{N}=18)$. Patients $(\mathrm{N}=63)$ who have treated with prophylactic FVIII were also assigned to receive emicizumab prophylaxis. The ABR of treated bleeding episodes was reduced to 1.5 (97\% reduction) in weekly treated patients and 1.3 (96\% reduction) in bi-weekly treated patients compared to those in nontreated patients at ABR 38.2. Onceweekly dosing of emicizumab reduced the ABR of treated bleeding episode by $68 \%$ (1.5 with emicizumab treatment vs 4.8 with prior FVIII prophylaxis), compared to their prior FVIII prophylaxis. In once-weekly and bi-weekly patients, $56 \%$ and $60 \%$ of the patients experienced no treated bleeding episodes during the period of clinical trial, respectively, but $0 \%$ of the patients experienced in nontreated patients. Injection site reactions were observed in $25 \%$ of the patients.

HAVEN 4 (NCT03020160), a nonrandomized openlabel study, has been conducted for assessing emicizumab subcutaneous administered tetra-weekly to PwHA with and without inhibitors $(\mathrm{N}=48 ; \geq 12$ years old $){ }^{35}$ The study regimen was a loading dose of $3 \mathrm{mg} / \mathrm{kg} /$ week for four weeks, followed by emicizumab $6 \mathrm{mg} / \mathrm{kg}$ tetraweekly. An interim data demonstrated that tetra-weekly dosing of emicizumab at $6 \mathrm{mg} / \mathrm{kg}$ exhibited a PK pattern similar to the dosing regimen at $1.5 \mathrm{mg} / \mathrm{kg} /$ week. ABR of treated bleeding episodes and all bleeding episodes were 2.4 and 4.5 , respectively, and $56 \%$ and $29 \%$ were free of bleeding. No SAEs and no ADAs were observed.

HAVEN 3 and HAVEN 4 have demonstrated the clinically significant prophylactic effect and safety of emicizumab in adult or adolescent patients. HOHOEMI (JapicCTI173710) is a study initiated in Japan, aiming to examine emicizumab prophylaxis in 13 children $(<12$ years old) including PUPs without inhibitors to FVIII. ${ }^{36}$ Emicizumab has been administered at doses of either $3 \mathrm{mg} / \mathrm{kg}$ bi-weekly or $6 \mathrm{mg} / \mathrm{kg}$ tetra-weekly. In the interim data, 3 out of 6 participants in biweekly cohort and 5 out of 7 patients (including 1 PUP) in tetra-weekly cohort experienced no bleeding events. There were no treated spontaneous bleedings and a total of 6 treated bleeds were traumatic. Two out of them were treated joint bleedings. ABRs for bleeds treated with FVIII products under emicizumab prophylaxis were 1.6 and 1.0 in biweekly and tetra-weekly cohorts, respectively. No SAE or TMA/TE was reported.

\section{Patient selection}

\section{Patients with inhibitor}

PwHA with inhibitor suffer from severe bleeding, but the effect of hemostatic treatment by BPAs for the patients is not so much stable as FVIII concentrates for noninhibitor patients, resulting in high morbidity and mortality of PwHA with inhibitor. ${ }^{37-39}$ The excellent clinical effects by practice of emicizumab for PwHA with inhibitor have been reported in the phase $1 / 2$ and phase 3 (HAVEN 1, 2 and 4) clinical studies. There seems to be no objection to applying emicizumab as an alternate first-line therapy for any existing BPA as hemostatic treatment for patients in which inhibitors have already appeared. Concomitant therapy with BPAs is considered on breakthrough bleeds during emicizumab prophylaxis, but in particular, in combination with aPCC, TEs such as TMA/TE are 
reported, which requires caution. There are pros and cons about when it is desirable to introduce emicizumab for cases where inhibitors have just appeared because eradication of inhibitors by ITI therapy would be another option. It is also wondering how to introduce emicizumab for patients under ITI therapy.

\section{Noninhibitor severe patients}

As majorities of severe PwHA without inhibitor are under prophylaxis by $\mathrm{CFCs},{ }^{40}$ patient selection may be required for consideration on introduction of emicizumab prophylaxis for the patients. In the previouslytreated patients (PTPs) case, it is necessary to select adaptive cases from the viewpoints of whether the hemostatic effect can be retained by switching from CFC prophylaxis to emicizumab, what difference in adaptation by physical activity is expected, how to suppress the progress of arthropathy. Alternatively, in PUPs, it is necessary to carefully examine pros and cons accompanying introduction of emicizumab prophylaxis as the PUPs. In this section, we consider its adaptation for each stage of age in life.

\section{Newborn stage}

For PwHA, one of the biggest problems at an early stage after birth is how to avoid the occurrence of intracranial hemorrhage (ICH). The incidence of ICH in children with HA is high at $3.5-4.0 \%,{ }^{41-43}$ compared to that in healthy infants, and intensive FVIII use following emergency medical treatment after onset is associated with the occurrence of inhibitor. ${ }^{44}$ However, considering the PKs of subcutaneous emicizumab, ${ }^{32}$ it might not be expected to exert a hemostatic effect immediately even if administered at an early stage after birth. Therefore, from the viewpoint of avoiding $\mathrm{ICH}$, we should discuss deeply the early administration of emicizumab after birth, but in case of the need to prevent ICH-onset after instrument delivery, CFCs such as FVIII should be considered. There is no data on the efficacy and safety of emicizumab administration for the newborn with HA so far. The potentials of coagulation, anticoagulation and fibrinolysis in the newborn clearly differ with those in non-newborn. Therefore, regarding safety in newborns, the hemostatic laboratory research, even in vitro, should be required.

Infant to pre-school stage in infancy, bleeding frequency increases due to an increase in activity associated with normal growth and development of infants, trauma, and patients most often experience initial joint bleeding. Therefore, in response to an increase in the bleeding risk, this stage is generally the time to introduce prophylactic replacement therapy by CFCs for PwHA. Although infant data are poor in the emicizumab clinical trials so far, in consideration of that CFCs prophylaxis is generally started from once a week and a trough FVIII level at the initial stage of introduction of CFCs prophylaxis becomes almost a moderate level $(1<$ FVIII:C $<5 \mathrm{IU} / \mathrm{dL})$, it is feasible to obtain equal or higher hemostatic effect by emicizumab and replacing CFCs prophylaxis with emicizumab prophylaxis appears to be reasonable. Moreover, concerning the issues of peripheral blood access at this time and of venipuncture pain, there is advantage in emicizumab subcutaneously accessible. However, emicizumab is not suitable as an episodic hemostatic treatment for acute hemorrhage, unlike CFCs in which initial administration is usually after acute bleeding. Therefore, how to introduce emicizumab and how to set its optimal dose-regimen is requiresd to be argueddiscussion. Further accumulation of clinical data is required.

\section{School age}

In school age, the range of behavior outside the home is wider, it is important to suppress the breakthrough bleeds and to treat early even if the bleed has occurred, in order to perform highly active group life in a stable manner. Therefore, the importance of prophylaxis has increased, and many children with HA are able to acquire home selfinjection techniques. In many children with HA, switching from CFCs prophylaxis to emicizumab prophylaxis is reasonable, but for patients who become more active since school age, suppression of breakthrough bleeds may be difficult with emicizumab prophylaxis alone. It is necessary to further consider the setting of optimal dose and concomitant use with CFCs. In addition, attention must be paid to the progression of arthropathy, especially by repetition of articular bleeding.

\section{Adolescent stage}

In adolescence, the condition of the joints affects the effectiveness of emicizumab prophylaxis. In the phase 1 and 2 studies, ${ }^{22,23}$ annual joint bleeding rates (AjBR) decreased sharply in the subjects with good joint condition assigned to low-dose arm (emicizumab concentration $\sim 10-16 \mu \mathrm{g} / \mathrm{mL}$ ), whereas only a modest decrease in AjBR was observed in the subject with severe arthropathy even with higher dose-regimen (emicizumab concentration $80-100 \mu \mathrm{g} / \mathrm{mL}$ ). Therefore, in terms of introduction of emicizumab prophylaxis for young adults, it is important to evaluate the joint condition 
by checking the presence of target joints or by the MRI and/or X-ray image inspection.

\section{Older age}

Patients in older age require care for organ dysfunction, malignant tumors and arterial events associated with atherosclerosis, accompanying aging in addition to such problems as arthropathy in young adulthood. Because of difficulty of blood access due to their aging, self-infusion or caregiver-infusion of CFC prophylaxis might be hard. Furthermore, the physical activity at this age is usually modest compared to the adolescent stage, therefore, it is reasonable to introduce emicizumab prophylaxis. Hemostatic treatment should be selected in consideration of thrombotic complication, when invasive treatment is required.

In any age group, in patients with PTPs, it is necessary to periodically examine for anti-FVIII inhibitors. Alternatively, in PUPs, it is unknown whether the risk of inhibitor occurrence is similar or not between in the case where $\mathrm{CFC}$ prophylaxis is started at any stage of age and the case where CFC treatment is repeated for breakthrough bleeds after introduction of emicizumab prophylaxis at PUPs.

\section{Mild/moderate patients}

Mild/moderate PwHA with inhibitor is basically the same as the above severe PwHA with inhibitor. In previous clinical trials, it has been observed only for severe patients, and there is no data on the efficacy and safety of emicizumab administration for mild/moderate noninhibitor patients. However, there are cases where the joint disorder progresses even though there is no obvious joint bleeding in mild/moderate PwHA. ${ }^{45}$ Since it is also known that inhibitors are developed by intensive administration of CFCs in mild/moderate HA, emicizumab, theoretically with no risk of the occurrence of anti-FVIII inhibitors, is expected as a new hemostatic agent. Concomitant administration of CFCs with emicizumab prophylaxis or mild/ moderate PwHA with emicizumab prophylaxis in PUPs is to be argued from the viewpoint of safety. For moderate PwHA who should be a candidate for CFC prophylaxis because of spontaneous bleedings, emicizumab prophylaxis would also be acceptable in consideration of pharmacokinetic characteristics of emicizumab which renders much lower affinity to FIXa and FX compared to FVIIIa, ${ }^{20}$ but the lack of data needs to be considered. Moreover, it is necessary to consider the indications of emicizumab prophylaxis for mild PwHA from the viewpoint of frequency of bleeding and cost-effectiveness. The higher efficacy needs to be proven in mild PwHA before using emicizumab.

\section{Special consideration}

Serious adverse events (SAEs) - thromboembolic risk The most common AEs which emicizumab-treated patients experienced in the clinical study are injection site reactions such as erythema, hematoma and pruritus $(16.7-25 \%){ }^{22,23,33-36}$ All were mild or moderate in intensity and resolved. As SAEs, the serious thrombotic events such as threeTMA and two VTEs (sinus vein thrombosis and superficial thrombophlebitis) under emicizumab prophylaxis were recorded through the HAVEN 1 study. ${ }^{24,46}$ TMA occurred in a patient after a multiple treatment of aPCC for four days of rectal bleeding. The patient eventually died, although the TMA had disappeared by the time of death. All thromboembolic events occurred during treatment with aPCC against breakthrough bleeds and the risk of thrombosis was related to the dose and intensity of administration. All thrombotic events were observed during high-dose (over $100 \mathrm{U} / \mathrm{kg}$ / day) and long-time (for over 24 hours) aPCC therapy, whereas no SAE occurred when the use with low-dose of aPCC (less than $100 \mathrm{U} / \mathrm{kg} /$ day), shorter treatment periods (less than 24 hours), or the rFVIIa was used for breakthrough bleeds. Therefore, during emicizumab prophylaxis, treatment for breakthrough bleeds has been recommended to avoid use of aPCC as far as possible and use of rFVIIa or lowest dose approved BPAs. However, no TE was observed in PwHA without inhibitor-treated emicizumab when FVIII was concomitantly administered for hemostatic management at breakthrough bleeds through the clinical trials. However, in the postmarketing setting, additional four TEs had occurred without concomitant high dose of aPCC or with emicizumab use alone.

\section{Hemostatic monitoring}

Since emicizumab prophylaxis is highly effective in reducing bleeding episodes, the need for regularly monitoring of emicizumab treatment may not be essential under the general circumstances. However, consistent laboratory assessment should be established to monitor the hemostasis under emicizumab treatment in order to avoid the prothrombotic status in patients who had to be treated with FVIII or BPAs for breakthrough bleeds for surgical management, or ITI cases. Emicizumab does not require activation step by thrombin. 
The aPTT mediated by emicizumab shortens more excessively than that by FVIII. ${ }^{18,20}$ Consequently, emicizumab significantly influences on FVIII procoagulant activity and inhibitor titers, measured by aPTT-based one-stage clotting assay. Therefore, the hemostatic effect of emicizumab cannot be assessed by a one-stage clotting assay. So far we can measure the plasma concentration of this drug but we cannot judge the hemostatic efficacy by the plasma concentration. The clot waveform analysis ${ }^{47}$ and thrombin generation assay, ${ }^{48}$ known as the global coagulation assays, can assess the potential of coagulation function of emicizumab in PwHA. The usefulness of rotational thromboelastometry (NATEM mode) in whole blood in these circumstances has also been reported. ${ }^{49}$

Conventional aPTT-based, one-stage coagulation assays for the precise measurement of FVIII procoagulant activity and FVIII inhibitor titers in the presence of emicizumab have been establishedby utilizing the cancelling effects of two anti-idiotype antibodies against anti-FIX $\mathrm{F}\left(\mathrm{ab}^{\prime}\right)_{2}$ and anti$\mathrm{FX} \mathrm{F}\left(\mathrm{ab}^{\prime}\right)_{2}{ }^{50}$ However, chromogenic assays with bovinederived reagents are also becoming available commercially for these purposes. Advances with techniques of this nature for monitoring hemostasis during emicizumab prophylaxis could have a critical influence on the safe use.

\section{Perioperative hemostatic management}

The 29 surgical procedures in twenty-two patients with inhibitor undertaking emicizumab were reported in HAVEN 1 and HAVEN 2 studies (24 events in HAVEN 1, 5 events in HAVEN 2). ${ }^{51}$ Supplements of BPAs were employed for nine operations, whilst not for 20 operations. The procedures included six tooth extractions and nine CVAD-related procedures. Minor surgery was performed in 12 patients, and two patients undertaken the laparoscopic appendectomy and knee synovectomy. Prophylactic BPAs was used for nine procedures including eight cases for rFVIIa use (median $150 \mu \mathrm{g} / \mathrm{kg}$ ) and one case for aPCC use (49 U/kg). Postoperative bleeding was observed in seven out of 20 surgical procedures. Three patients were cured without prophylactic BPAs, and four were treated with BPAs. The patients under emicizumab prophylaxis could be successfully undertaken the majority of minor surgical procedure without prophylactic BPAs, although approximately $30 \%$ of cases required administration of BPA for controlling postoperative bleeding. In addition, concomitant tranexamic-acid therapy may be effective to prevent the bleeding for the patients under treatment by emicizumab on minor surgery.

\section{Antidrug antibody (ADA)}

A clinical study involving nonhemophilic volunteers with a single-injection has described that emicizumab-induced ADAs were detected in three out of 48 subjects (2/36; subcutaneously, $1 / 12$; intravenously), suggestive of a prevalence of $\sim 5 \%$ for subcutaneous administration. ${ }^{36}$ In the phase $1 / 2$ clinical study for PwHA, the emicizumab-induced ADAs were detected in four out of 18 patients $(22.2 \%)$ and ADAs in all patients were non-neutralizing antibodies. ${ }^{22,23}$ In the phase 3 clinical study, emicizumab-induced ADAs were detected in 14 out of 398 patients $(3.5 \%) .^{24,33-35}$ Three out of 14 ADA-positive patients developed the neutralizing antibodies. Among them, one patient had developed the complete disappearance of emicizumab from his circulating blood and had increases of bleeding episodes. Consequently the emicizumab treatment was discontinued in this patient. It would be necessary to pay attention to the development of ADA during the emicizumab treatment.

\section{Consideration of ITI}

In order to eradicate inhibitors, ITI is a unique approach widely accepted so far, in spite of being highly intensive, requiring frequent injection of CFCs often with CVAD in children because of difficulty in blood access, as described above (see "Introduction"). Emicizumab prophylaxis has the potential to reduce the necessity of ITI. The eradication of inhibitors remains an important option for eg, in surgical procedures. It remains controversial whether emicizumab should be administrated after eradication of inhibitor by ITI or solely prescribed independent of ITI for PwHA with inhibitor. Young ${ }^{52}$ pointed out the significance of eradication of inhibitor prior to introduction of emicizumab focusing on the burden such as higher mortality due to severe bleeding and potential risk of exclusion from future gene therapy for PwHA with inhibitor. In contrast, Le Quellec et al ${ }^{53}$ described that emicizumab which has potential benefit of subcutaneously available prophylaxis for breakthrough bleeds during ITI could be prescribed independent of ITI because emicizumab renders excellent hemostatic effect compared to BPA prophylaxis and has good safety profiles. Santagostino et $\mathrm{al}^{54}$ recommended at least one round of ITI because successful eradication of an inhibitor may allow more and safer therapeutic options for the patient in the future to prevent bleeding(eg, gene therapy) and to treat bleeds (with FVIII replacement rather than BPA). Further clinical evidence are required as for the potential use of emicizumab in the context of ITI. 


\section{Future consideration}

Taking into consideration the results from phase 1 and 2 studies that breakthrough bleeds have been well tolerated in the low dose cohort, ${ }^{22,32}$ even lower plasma concentration of emicizumab $(\sim 10-30 \mu \mathrm{g} / \mathrm{mL})$ than that $(\sim 50 \mu \mathrm{g} / \mathrm{mL})$ by current approved regimen could be effective as prophylaxis for PwHA whose articular condition has been fine irrespective of inhibitors. ${ }^{24,33,34,37}$ The licensed dose of emicizumab does not allow for dose escalation now, but the low dose of this drug may be safe in comparison with its standard dose on concomitant therapy with other hemostatic agents such as FVIII and rFVIIa for breakthrough bleeds during emicizumab prophylaxis. Alternatively, a higher dose of emicizumab than the licensed dose may also be effective for active patients with trouble in their joint health, but the lack of data, especially concerning safety and cost, needs to be considered.

\section{Conclusion}

The nature and development of emicizumab for PwHA irrespective of inhibitor have been outlined, and the outcomes of recent clinical trials have been reviewed. Moreover, patient selection and special consideration for use of emicizumab has been addressed. Further studies are needed, in order to accumulate conclusive clinical data on safety, especially regarding the risk of TMA/TE with the concomitant use of BPAs or FVIII, and in addition, longer-term use. Standardized techniques to monitor hemostasis in those patients receiving emicizumab will assist clinical management in these circumstances. The new era has just started with the novel hemostatic agent emicizumab and further clinical evidence is required for better treatment for PwHA in the future.

\section{Author contributions}

All authors contributed to data analysis, drafting and revising the article, gave final approval of the version to be published, and agree to be accountable for all aspects of the work.

\section{Disclosure}

$\mathrm{KN}$ is an inventor of the patents relating to emicizumab. $\mathrm{KY}$ and $\mathrm{KN}$ are engaged in clinical studies sponsored by Chugai and F. Hoffmann-La Roche. KY teaches a course endowed by Shire Japan Co. Ltd. KY also reports nonfinancial support from Chugai Pharmaceutical, F. Hoffmann-La Roche, and Shire Japan Co. Ltd., during the conduct of the study. KN reports grants and personal fees, from Shire, Japan Co. Ltd., Novo Nordisk, Bioverativ, and Bayer, during the conduct of the study. $\mathrm{KN}$ also receives personal fees from Chugai Pharmaceutical. In addition, $\mathrm{KN}$ has a patent pending with Chugai. The authors report no other conflicts of interest in this work.

\section{References}

1. White GC II, Rosendaal F, Aledort LM, Lusher JM, Rothschild C, Ingerslev J. Definitions in hemophilia: recommendation of the scientific subcommittee on factor VIII and factor IX of the scientific and standardization committee of the International Society on Thrombosis and Haemostasis. Thromb Haemost. 2001;85(3):560. doi:10.1055/s-00371615621

2. Luck JV Jr, Silva M, Rodriguez-Merchan EC, Ghalambor N, Zahiri CA, Finn RS. Hemophilic arthropathy. J Am Acad Orthop Surg. 2004;12(4):234-245.

3. Ferreira AA, Leite IC, Bustamante-Teixeira MT, et al. Health-related quality of life in hemophilia: results of the Hemophilia-Specific Quality of Life Index. (Haem-a-Qol) at a Brazilian blood center. Rev Bras Hematol Hemoter. 2013;35(5):314-318. doi:10.5581/15168484.20130108

4. Nilsson IM, Hedner U, Ahlberg A. Hemophilia prophylaxis in Sweden. Acta Paediatr Scand. 1976;65(2):129-135.

5. Manco-Johnson MJ, Abshire TC, Shapiro AD, et al. Prophylaxis versus episodic treatment to prevent joint disease in boys with severe hemophilia. N Engl J Med. 2007;357(6):535-544. doi:10.1056/NEJMoa067659

6. De Moerloose P, Urbancik W, Van Den Berg HM, et al. A survey of adherence to haemophilia therapy in six European countries: results and recommendations. Haemophilia. 2008;14(5):931-938. doi:10.1111/ j.1365-2516.2008.01843.x

7. van Os SB, Troop NA, Sullivan KR, Hart DP. Adherence to prophylaxis in adolescents and young adults with severe haemophilia: a quantitative study with patients. PLoS One. 2017;12(1):e0169880. doi:10.1371/journal.pone.0169880

8. KyngAÈ s HA, Kroll T, Duffy ME. Compliance in adolescents with chronic diseases: a review. J Adolesc Health. 2000;26(6):379-388.

9. Mahlangu J, Powell JS, Ragni MV, et al. Phase 3 study of recombinant factor VIII Fc fusion protein in severe hemophilia A. Blood. 2014;123(3):317-325. doi:10.1182/blood-2013-10-529974

10. Coyle TE, Reding MT, Lin JC, Michaels LA, Shah A, Powell J. Phase I study of BAY 94-9027, a PEGylated B-domain-deleted recombinant factor VIII with an extended half-life, in subjects with hemophilia A. J Thromb Haemost. 2014;12(4):488-496.

11. Turecek PL, Bossard MJ, Graninger M, et al. BAX 855, a PEGylated rFVIII product with prolonged half-life. Development, functional and structural characterisation. Hamostaseologie. 2012;32(Suppl 1):S29-38.

12. Stennicke HR, Kjalke M, Karpf DM, et al. A novel B-domain Oglyco PEGylated FVIII (N8-GP) demonstrates full efficacy and prolonged effect in hemophilic mice models. Blood. 2013;121(11):21082116. doi:10.1182/blood-2012-01-407494

13. Coppola A, Franchini M, Castaman G, et al. Treatment regimens with bypassing agents in patients with hemophilia A and inhibitors: a survey from the Italian Association of Hemophilia Centers (AICE). Semin Thromb Hemost. 2018;44(6):551-560. doi:10.1055/s-0038-1648230

14. Walsh CE, Jiménez-Yuste V, Auerswald G, et al. The burden of inhibitors in haemophilia patients. Thromb Haemost. 2016;116 (Suppl 1):S10-S17. doi:10.1160/TH16-01-0049

15. Hay CR, DiMichele DM; International Immune Tolerance Study. The principal results of the International Immune Tolerance Study: a randomized dose comparison. Blood. 2012;119(6):1335-1344. doi:10.1182/blood-2011-08-369132

16. Leissinger C, Gringeri A, Antmen B, et al. Anti-inhibitor coagulant complex prophylaxis in hemophilia with inhibitors. $N$ Engl J Med. 2011;365(18):1684-1692. doi:10.1056/NEJMoa1104435 
17. Rodriguez V, Mancuso ME, Warad D, et al. Central venous access device (CVAD) complications in Haemophilia with inhibitors undergoing immune tolerance induction: lessons from the international immune tolerance study. Haemophilia. 2015;21(5):e369-e374. doi:10.1111/hae.12740

18. Kitazawa T, Igawa T, Sampei Z, et al. A bispecific antibody to factors IXa and $\mathrm{X}$ restores factor VIII hemostatic activity in a hemophilia A model. Nat Med. 2012;18(10):1570-1574. doi:10.1038/nm.2942

19. Sampei Z, Igawa T, Soeda T, et al. Identification and multidimensional optimization of an asymmetric bispecific IgG antibody mimicking the function of factor VIII cofactor activity. PLoS One. 2013;8(2):e57479. doi:10.1371/journal.pone.0057479

20. Kitazawa T, Esaki K, Tachibana T, et al. Factor VIIIa-mimetic cofactor activity of a bispecific antibody to factors IX/IXa and $\mathrm{X} / \mathrm{Xa}$, emicizumab, depends on its ability to bridge the antigens. Thromb Haemost. 2017;117(7):1348-1357. doi:10.1160/TH17-01-0030

21. Uchida N, Sambe T, Yoneyama K, et al. A first-in-human phase 1 study of ACE910, a novel factor VIII-mimetic bispecific antibody, in healthy subjects. Blood. 2016;127(13):1633-1641. doi:10.1182/ blood-2015-06-650226

22. Shima M, Hanabusa H, Taki M, et al. Factor VIII-mimetic function of humanized bispecific antibody in hemophilia A. $N$ Engl J Med. 2016;374(21):2044-2053. doi:10.1056/NEJMoa1511769

23. Shima M, Hanabusa H, Taki M, et al. Long-term safety and efficacy of emicizumab in a phase $1 / 2$ study in patients with hemophilia A with or without inhibitors. Blood Adv. 2017;1(22):1891-1899. doi:10.1182/bloodadvances.2017006684

24. Oldenburg J, Mahlangu JN, Kim B, et al. Emicizumab prophylaxis in hemophilia A with inhibitors. N Engl J Med. 2017;377(9):809-818. doi:10.1056/NEJMoa1703068

25. Chowdary P, Lethagen S, Friedrich U, et al. Safety and pharmacokinetics of anti-TFPI antibody (concizumab) in healthy volunteers and patients with hemophilia: a randomized first human dose trial. $J$ Thromb Haemost. 2015;13(5):743-754. doi:10.1111/jth.12864

26. Eichler H, Angchaisuksiri P, Kavakli K, et al. A randomized trial of safety, pharmacokinetics and pharmacodynamics of concizumab in people with hemophilia A. J Thromb Haemost. 2018;16(11):21842195. doi:10.1111/jth.14272

27. Pasi KJ, Rangarajan S, Georgiev P, et al. Targeting of antithrombin in hemophilia A or B with RNAi therapy. N Engl J Med. 2017;377 (9):819-828. doi:10.1056/NEJMoa1616569

28. Fay PJ. Regulation of factor VIIIa in the intrinsic factor Xase. Thromb Haemost. 1999;82(2):193-200.

29. Minami H, Nogami K, Soeda T, Kitazawa T, Hattori K, Shima M. The factor VIII heavy chain improves emicizumab-tenase assembly to enhance the factor VIII-mimicking cofactor activity. Thromb Res. 2018;166:77-79. doi:10.1016/j.thromres.2018.04.016

30. Yada K, Nogami K, Shinozawa K, et al. Emicizumab-mediated hemostatic function in patients with hemophilia $\mathrm{A}$ is down regulated by activated protein $\mathrm{C}$ through inactivation of activated factor $\mathrm{V}$. $\mathrm{Br} J$ Haematol. 2018;183(2):257-266. doi:10.1111/bjh.15525

31. Noguchi-Sasaki M, Soeda T, Ueyama A, et al. Emicizumab, a bispecific antibody to factors IX/IXa and X/Xa, does not interfere with antithrombin or TFPI activity in vitro. TH Open. 2018;2(1):e96e103. doi:10.1055/s-0038-1636538

32. Yoneyama K, Schmitt C, Kotani N, et al. A pharmacometric approach to substitute for a conventional dose-finding study in rare diseases: example of phase III dose selection for emicizumab in hemophilia A. Clin Pharmacokinet. 2018;57(9):1123-1134. doi:10.1007/s40262017-0616-3

33. Young G, Liesner R, Sidonio RF, et al. Emicizumab prophylaxis provides flexible and effective bleed control in children with hemophilia $\alpha$ with inhibitors: results from the HAVEN 2 study. Blood. 2018;132:632.

34. Mahlangu J, Oldenburg J, Paz-Priel I, et al. Emicizumab prophylaxis in patients who have hemophilia A without inhibitors. $N$ Engl J Med. 2018;379(9):811-822. doi:10.1056/NEJMoa1803550
35. Pipe SW, Shima M, Lehle M, et al. Efficacy, safety, and pharmacokinetics of emicizumab prophylaxis given every 4 weeks in people with haemophilia A (HAVEN 4): a multicentre, open-label, nonrandomised phase 3 study. Lancet Haematol. 2019. [Epub ahead of print]. doi:10.1016/S2352-3026(19)30054-7

36. Shima M, Nogami K, Nagami S, et al. Every 2 weeks or every 4 weeks subcutaneous injection of emicizumab in pediatric patients with severe hemophilia a without inhibitors: a multi-center, openlabel study in Japan (HOHOEMI study). Blood. 2018;132:1186.

37. Morfini M, Haya S, Tagariello G, et al. European study on orthopaedic status of haemophilia patients with inhibitors. Haemophilia. 2007;13(5):606-612. doi:10.1111/j.1365-2516.2007.01518.x

38. Monahan PE, Baker JR, Riske B, Soucie JM. Physical functioning in boys with hemophilia in the U.S. Am J Prev Med. 2011;41(6 suppl 4): S360-368. doi:10.1016/j.amepre.2011.09.017

39. Walsh CE, Soucie JM, Miller CH; United States Hemophilia Treatment Center Network. Impact of inhibitors on hemophilia A mortality in the United States. Am J Hematol. 2015;90(5):400-405. doi:10.1002/ajh.23957

40. Biss TT, Chan AK, Blanchette VS, Iwenofu LN, McLimont M, Carcao MD; Association of Hemophilia ClinicDirectors of Canada (AHCDC) Canadian Association of Nurses in Hemophilia Care (CANHC). Theuse of prophylaxis in 2663 children and adults with haemophilia: results of the 2006 Canadian national haemophilia prophylaxis survey. Haemophilia. 2008;14(5):923-930. doi:10.1111/ j.1365-2516.2008.01810.x

41. Ljung R, Lindgren AC, Petrini P, Tengborn L. Normal vaginal delivery is to be recommended for haemophilia carrier gravidae. Acta Paediatr. 1994;83(6):609-611.

42. Klinge J, Auberger K, Auerswald G, Brackmann HH, Mauz-Körholz C, Kreuz W. Prevalence and outcome of intracranial haemorrhage in haemophiliacs - a survey of the paediatric group of the German Society of Thrombosis and Haemostasis (GTH). Eur J Pediatr. 1999;158(Suppl 3):S162-S165.

43. Revel-Vilk S, Golomb MR, Achonu C, et al. Effect of intracranial bleeds on the health and quality of life of boys with hemophilia. J Pediatr. 2004;144(4):490-495. doi:10.1016/j.jpeds.2003.12.016

44. Garagiola I, Palla R, Peyvandi F. Risk factors for inhibitor development in severe hemophilia A. Thromb Res. 2018;168:20-27. doi:10.1016/j.thromres.2018.05.027

45. Di Minno MN, Ambrosino P, Franchini M, Coppola A, Di Minno G. Arthropathy inpatients with moderate hemophilia a: a systematic review of the literature. Semin Thromb Hemost. 2013;39(7):723731. doi:10.1055/s-0033-1354422

46. Tiede A. Thromboembolic risks of non-factor replacement therapies in hemophilia. Hamostaseologie. 2017;37(4):307-310. doi:10.5482/20170004

47. Nogami K, Matsumoto T, Tabuchi Y, et al. Modified clot waveform analysis to measure plasma coagulation potential in the presence of the anti-factor IXa/factor $\mathrm{X}$ bispecific antibody emicizumab. $J$ Thromb Haemost. 2018;16(6):1078-1088.

48. Dargaud Y, Lienhart A, Janbain M, et al. Use of thrombin generation assay to personalize treatment of breakthrough bleeds in a patient with hemophilia and inhibitors receiving prophylaxis with emicizumab. Haematologica. 2018;103(4):e181-e183. doi:10.3324/ haematol.2017.185330

49. Yada K, Nogami K, Kitazawa T, et al. Mode of enhancement in the global hemostatic potentials with concomitant use of bypassing agents and emicizumab in hemophilia A patients with inhibitor evaluated by ROTEM. Res Pract Thromb Haemost. 2017;1(Suppl 1):163-164.

50. Nogami K, Soeda T, Matsumoto T, Kawabe Y, Kitazawa T, Shima M. Routine measurements of factor VIII activity and inhibitor titer in the presence of emicizumab utilizing anti-idiotype monoclonal antibodies. J Thromb Haemost. 2018;16 (7):1383-1390. doi:10.1111/jth.14135 
51. Kruse-Jarres R, Callaghan MU, Croteau SE, et al. Surgical experience in two multicenter, open-label phase 3 studies of emicizumab in persons with hemophilia A with inhibitors (HAVEN 1 and HAVEN 2). Blood. 2017;130(Suppl):89.

52. Young G. Implementing emicizumab in hemophilia inhibitor management: emicizumab should be prescribed after tolerance. Blood Adv. 2018;2(20):2780-2782. doi:10.1182/bloodadvances.2018015842
53. Le Quellec S, Negrier C. Emicizumab should be prescribed independent of immune tolerance induction. Blood Adv. 2018;2(20):27832786. doi:10.1182/bloodadvances.2018015859

54. Santagostino E, Young G, Escuriola Ettingshausen C, Jimenez-Yuste V, Carcao M. Inhibitors: a need for eradication? Acta Haematol. 2019;141(3):151-155. doi:10.1159/000495454

\section{Publish your work in this journal}

The Journal of Blood Medicine is an international, peer-reviewed, open access, online journal publishing laboratory, experimental and clinical aspects of all aspect pertaining to blood based medicine including but not limited to: Transfusion Medicine; Blood collection, Donor issues, Transmittable diseases, and Blood banking logistics; Immunohematology; Artificial and alternative blood based therapeutics; Hematology; Biotechnology/nanotechnology of blood related medicine; Legal aspects of blood medicine; Historical perspectives. The manuscript management system is completely online and includes a very quick and fair peer-review system. Visit http://www.dovepress.com/testimonials.php to read real quotes from published authors. 